Original Research Paper

\title{
Smart Orthopaedic Implants: Applications in Total Knee Arthroplasty
}

\author{
${ }^{1}$ Matthew K. Dion, ${ }^{1}$ John Drazan, ${ }^{1}$ Khaled Abdoun, ${ }^{1}$ Sarah Giddings, \\ ${ }^{2}$ Vishal Desai, ${ }^{2}$ Nathaniel C. Cady, ${ }^{3}$ Reena Dahle, ${ }^{4}$ Jared T. Roberts and ${ }^{1}$ Eric H. Ledet \\ ${ }^{I}$ Department of Biomedical Engineering, Rensselaer Polytechnic Institute, Troy, NY, USA \\ ${ }^{2}$ College of Nanoscale Science and Engineering, SUNY Polytechnic Institute, Albany, NY, USA \\ ${ }^{3}$ Department of Electrical Engineering, SUNY New Paltz, New Paltz, NY, USA \\ ${ }^{4}$ Capital Region Orthopaedic Group, Albany, NY, USA
}

Article history

Received: 01-12-2016

Revised: 02-12-2016

Accepted: 23-12-2016

Corresponding Author:

Eric H. Ledet

Department of Biomedical

Engineering, Rensselaer

Polytechnic Institute, Troy,

NY, USA

Email: ledete@rpi.edu

\begin{abstract}
Total knee arthroplasty is a common orthopaedic procedure conducted in the United States with approximately 700,000 surgeries performed annually. A common complication following total knee arthroplasty is anterior knee pain which affects tens to hundreds of thousands of people each year. The exact mechanism that leads to anterior knee pain remains unknown, but improper component selection may cause pathologic loading of the knee which leads to pain. Measuring loads in the knee to elucidate the mechanisms underlying anterior knee pain remains a challenge because the joints are so small. Using novel wireless sensor technology, we have developed and validated the first "smart" patellar implant capable of measuring force magnitude and force distribution in the knee. Implantable force sensors were calibrated and tested through the range of physiologic loading. Three sensors were then interfaced with a Zimmer patellar implant and placed into a custom loading apparatus. The smart patellar implant was then incrementally loaded from 0-500 N. Sensor signals were all recorded simultaneously in real time to measure the load across the patellofemoral joint. Results demonstrated that the smart patellar implant was able to accurately measure the load being transmitted across the simulated patellofemoral joint.
\end{abstract}

Keywords: Total Knee Arthroplasty, Anterior Knee Pain, Smart Patellar Implant, Orthopaedics

\section{Introduction}

Osteoarthritis (OA) is a leading cause of chronic disability and pain. For patients who fail conservative care, the gold standard treatment for knee OA is a Total Knee Arthroplasty (TKA). During TKA, the pathologic tissues are replaced with multi-component implants. In 2010, the total costs associated with TKA procedures was over $\$ 11$ billion in the United States alone (Losina et al., 2009). The total number of TKA procedures performed annually is expected to rise $673 \%$ from 600,000 to 3.48 million by the year 2030 (Kurtz et al., 2007). This is primarily due to an increasing life expectancy and increasing elderly population in the United States. Postoperatively, chronic anterior knee pain is a common complication following TKA. The incidence of anterior knee pain following TKA varies from study to study, but is commonly reported to affect $8-21 \%$ of TKA patients annually in the
US (Sensi et al., 2011; Zou et al., 2011). Anterior knee pain post-TKA has been reported at rates as high as 53.8\% (Metsna et al., 2014).

Analysis of clinical and experimental in vitro data has led to the identification of a number of factors that increase a patient's risk of anterior knee pain and factors that contribute to the mechanism that causes it. These factors can be divided into functional (muscle imbalance, varus/valgus knees) and mechanical (improper positioning of prosthesis, component loosening, patellar fracture); however, the exact etiology of anterior knee pain following TKA remains controversial and there is no established mechanism for why or exactly how these factors influence the joint in vivo (Petersen et al., 2014). Anecdotal evidence suggests that improperly sized implants alter the biomechanics of the joint thus leading to increased forces and anterior knee pain. 
Models and in vitro experimental data have been used to predict in vivo forces in the patellofemoral joint of the knee, but there is very limited in vivo data to validate these models. This is because there remain major hurdles to in vivo experimental data collection from the patellofemoral joint. Most significant are the small joint space, articulating motion and complex geometry. To date, no force sensing technology has been able to facilitate capture of dynamic real time in vivo forces in the patellofemoral joint. Yet, measurement of patellofemoral forces could contribute significantly to the understanding of patellofemoral joint mechanics and the etiology of anterior knee pain.

Traditional force sensing technologies, such as wired load cells and strain gauges, are too large for the small patellofemoral space and require power, signal conditioning and telemetry. The small joint space and articulating nature of the patellofemoral joint make it impossible for these force sensing technologies to be interfaced with implants to allow for in vivo force measurement. The purpose of this study was to utilize a novel force sensing technology to develop and test the first "smart" patella.

\section{Materials and Methods}

\section{Novel Sensor Technology}

We have developed a simple, inexpensive, wireless, battery-less, telemetry-less sensor technology which requires no onboard electronics and has no electrical connections (Drazan et al., 2014; Wachs et al., 2013). These simple force sensors are comprised simply of two, anti-aligned Archimedean spiral coils separated by a solid intervening dielectric layer. These spiral coils are fabricated on wafers out of conductive materials such as gold, platinum or copper, with copper prototypes used for experimental testing. The sensors are simple lumped parameter inductorcapacitor (LC) passive resonators. As with a parallel plate capacitor, when the distance between two coils changes, there is a shift in the system capacitance which results in a change of the sensor resonant frequency. Equation 1 shows the general relationship between sensor deformation and resonant frequency. This resonant frequency is measured wirelessly in real time by tracking the S11 return loss parameter with a network analyzer and custom fabricated coil antenna. Once calibrated, the resonant frequency is used to determine the forces applied to each sensor:

$$
\Delta f=\frac{1}{2 \pi \sqrt{L \frac{\epsilon_{r} \in_{o} A_{C}}{\Delta l}}}
$$

Where:

$C=$ Capacitance $=\frac{\epsilon_{r} \in_{o} A_{C}}{\Delta l}$

$\epsilon_{r}=$ Relative permittivity of intervening layer

$\epsilon_{o}=$ Dielectric constant of free space

$A_{C}=$ Area of Conductive Traces

$\Delta l=$ Distance between Two Coils

$L=$ Inductance of a Sensor

$\Delta f=$ Sensor Resonant Frequency where, $f=\frac{1}{2 \pi \sqrt{L C}}$

\section{Sensor Fabrication}

For testing, halo shaped copper Archimedean spiral coils (Fig. 1A) were fabricated on Borofloat 33 glass wafers using contact photolithography in combination with $\mathrm{Cu}$ electroplating. Briefly, wafers were coated with a $\mathrm{Ni}$ metal seed layer, followed by photoresist deposition and patterning (via contact lithography) such that multiple coils could be fabricated on each glass wafer. Following copper electroplating, the photoresist and exposed $\mathrm{Ni}$ seed layer were removed, resulting in free standing $\mathrm{Cu}$ coil structures. Following fabrication, the wafers were diced, as shown in Fig. 1B. The center of each coil was then drilled (Valley Design Corp, Shirley, Ma). Coils were then cleaned using oxygen plasma and coated with silane A-174, an adhesion promoter. The individual coils were then coated with $10 \mu \mathrm{m}$ of parylene C. Individual coils were then paired with a complimentary coil and bound using a die binder (Finetech, Manchester, NH) in an anti-aligned configuration.

Sensors (paired bound coils on wafers) were comprised of a uniform $20 \mu \mathrm{m}$ intervening layer of parylene $\mathrm{C}$, as shown in Fig. 1C. The final dimensions of the sensors were inner diameter $5.2 \mathrm{~mm}$, outer diameter $11.2 \mathrm{~mm}$ and total thickness $2.02 \mathrm{~mm}(1 \mathrm{~mm}$ glass on each side of coil and $20 \mu \mathrm{m}$ intervening layer). The geometry of the sensors was specifically chosen to interface with an existing patellar implant.

\section{Sensor Mechanical Testing}

Once the sensors were assembled, they were calibrated. Sensors were individually loaded in a mechanical testing system (MTS Systems, Eden Prairie, MN) by incrementally applying forces to each sensor from 0-300 $\mathrm{N}$ in axial compression. Simultaneously, the resonant frequency of each sensor was recorded in real time. The resonant frequency values were used to create a calibration curve specific to each sensor. The loading protocol was repeated 5 times for each sensor to ensure the repeatability of the calibration. 
Once calibrated, three sensors were arranged in a planar triangular configuration in a manner mimicking the posts on the anterior surface of a patellar implant (Fig. 2B). The three sensor array was loaded simultaneously (in parallel) from $0-500 \mathrm{~N}$ while resonant frequencies of all three sensors were recorded simultaneously and in real time. This testing was repeated 3 times. The applied load was compared to the measured load using each sensor's calibration data.

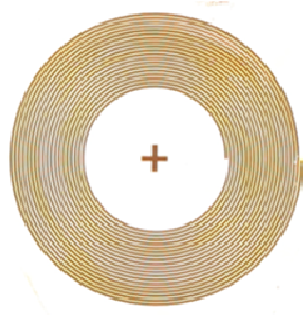

A

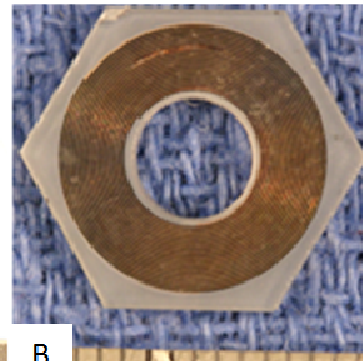

B

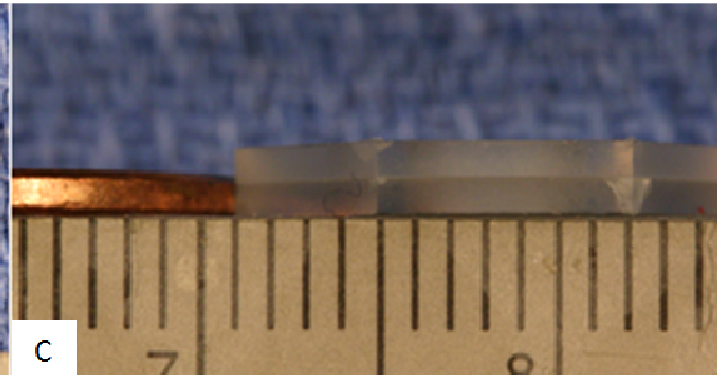

Fig. 1. (A) Individual halo coils were cleaned and coated with silane. (B) Halo sensors were drilled in their inner diameter. (C) Coils were bonded to form sensors with a $20 \mu \mathrm{m}$ intervening layer of Parylene $\mathrm{C}$ (penny shown for scale)

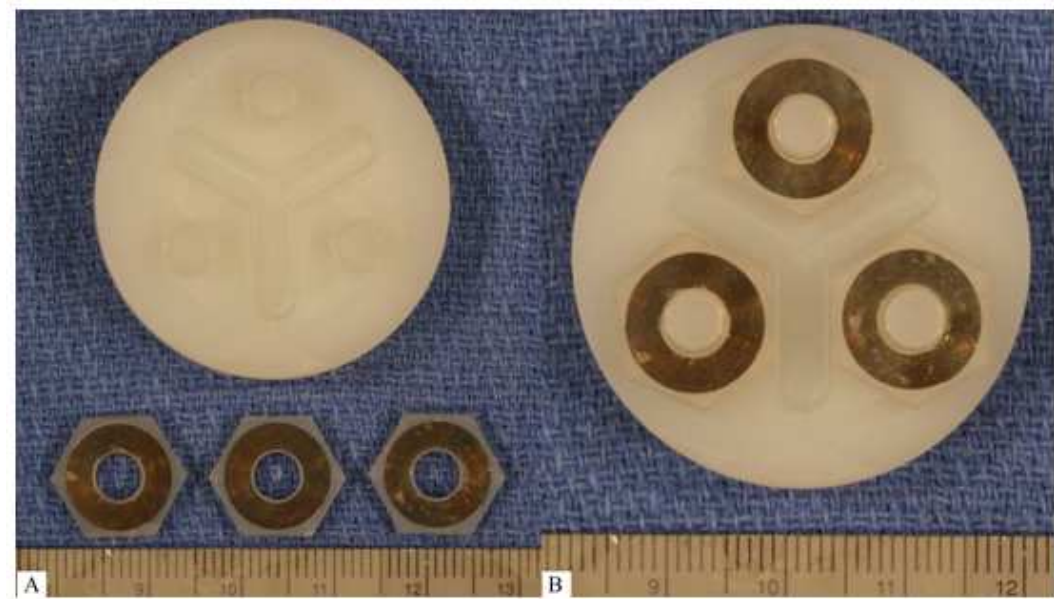

Fig. 2. (A) Patellar implant and 3 halo sensors. (B) Sensors were interfaced with the Zimmer implant with no modifications required

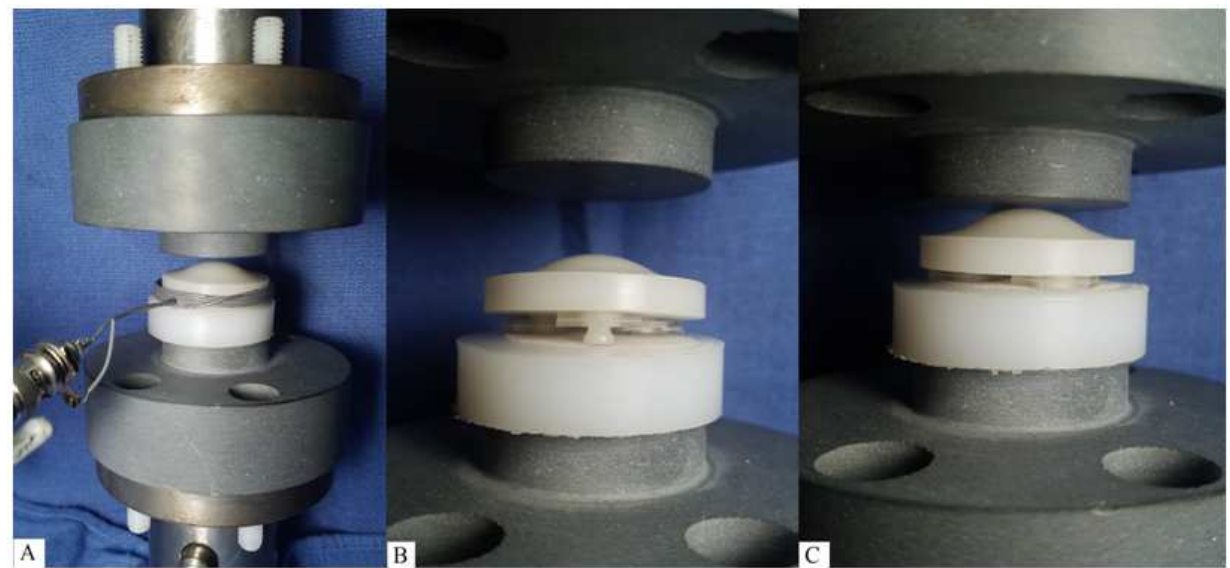

Fig. 3. (A) The smart patellar implant was placed within a custom fixture and the antenna placed around the implant for testing. (B) Sensors were placed around the posts in the patella to make a smart implant. (C) The assembled smart patellar implant was placed in the testing machine for mechanical testing 
The three sensors were then interfaced with an off-ofthe-shelf $8.5 \mathrm{~mm}$ patellar implant (Zimmer, Warsaw, IN) by placing the sensors over the three posts on the anterior surface, as shown in Fig. 2. Interfacing the sensors with the patellar implant required no modification to the implant. Once interfaced with the patellar implant, the smart patella was placed into a custom fixture in the mechanical testing machine, as shown in Fig. 3. The patella was loaded from 0-500 N. Sensor resonant frequencies were recorded simultaneously and used to calculate individual loads. These loads were then summed to obtain the total load across the patellar implant. Applied load, measured using a wired load cell held in series with the patella, was compared to forces measured using the smart patella.

\section{Results}

A characteristic sensor calibration curve is shown in Fig. 4. The sensor response to load was highly linear with a $\mathrm{R}^{2}=0.9982$.

When three sensors were loaded in the planar triangular configuration, the measured loads were in good agreement (slope $=0.9426, \mathrm{R}^{2}=0.9805$ ) with applied load as shown in Fig. 5.

Fig. 6 shows the results of four replicates in which the patellar button was loaded and then allowed to rest for 5 minutes before the next load was applied. The mean values of these four replicates are shown in Fig. 7. There is a good correlation between measured and applied load (slope $=1.101$ and $\mathrm{R}^{2}=0.9822$ ).

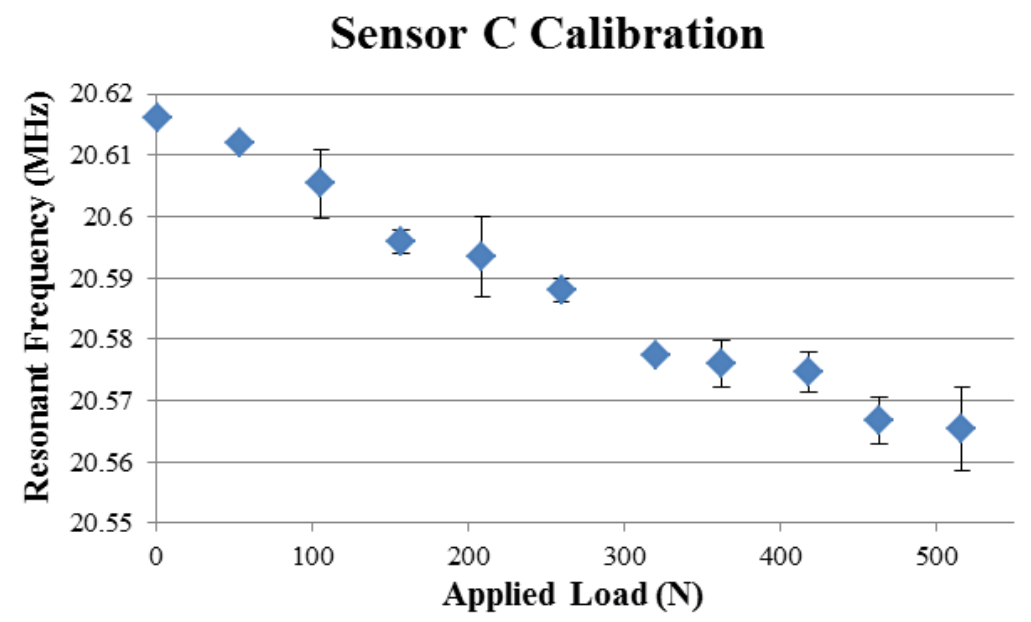

Fig. 4. Calibration curves were generated from stepwise compressive loading of a sensor through five replicates. Error bars are one standard deviation. $\left(\mathrm{R}^{2}=0.9802\right)$

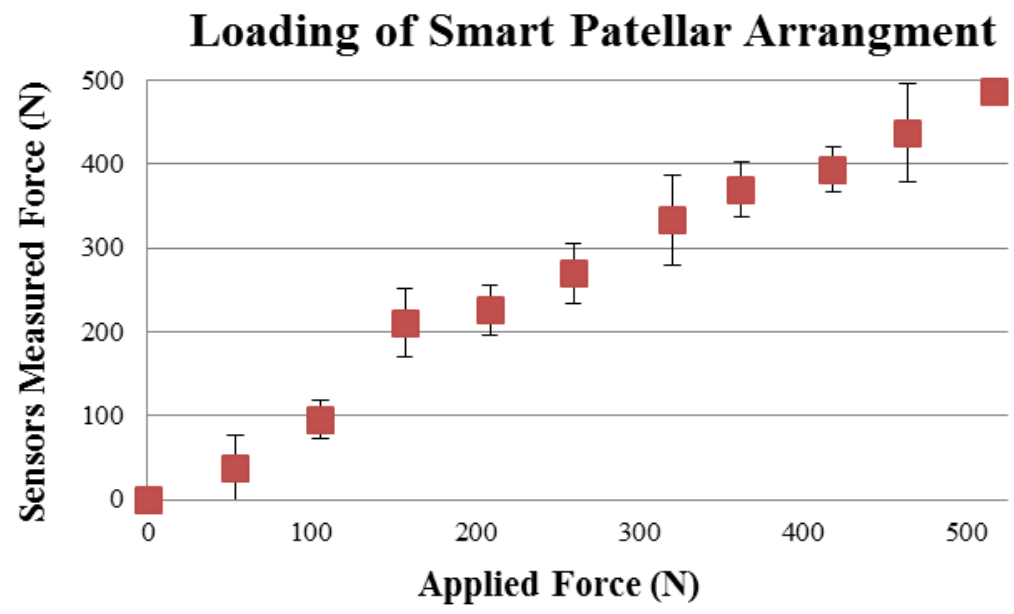

Fig. 5. The smart patella was loaded through three compressive cycles $\left(\mathrm{R}^{2}=0.9805\right)$. Error bars represent one standard deviation 


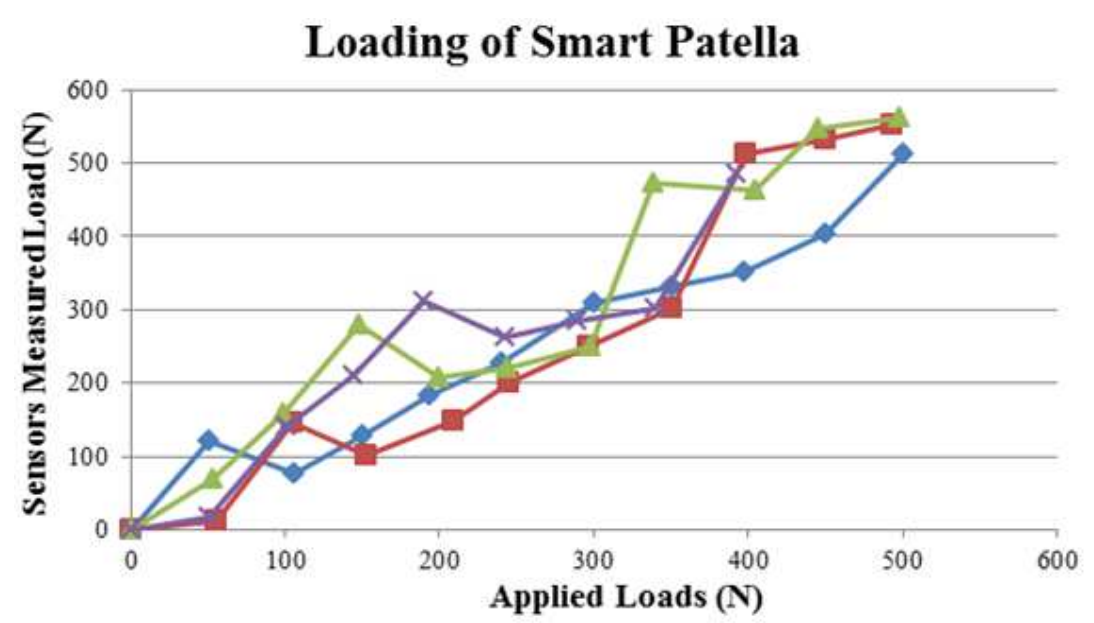

Fig. 6. The smart patella was loaded through four replicates. Individual sensor forces were summed to obtain total load.

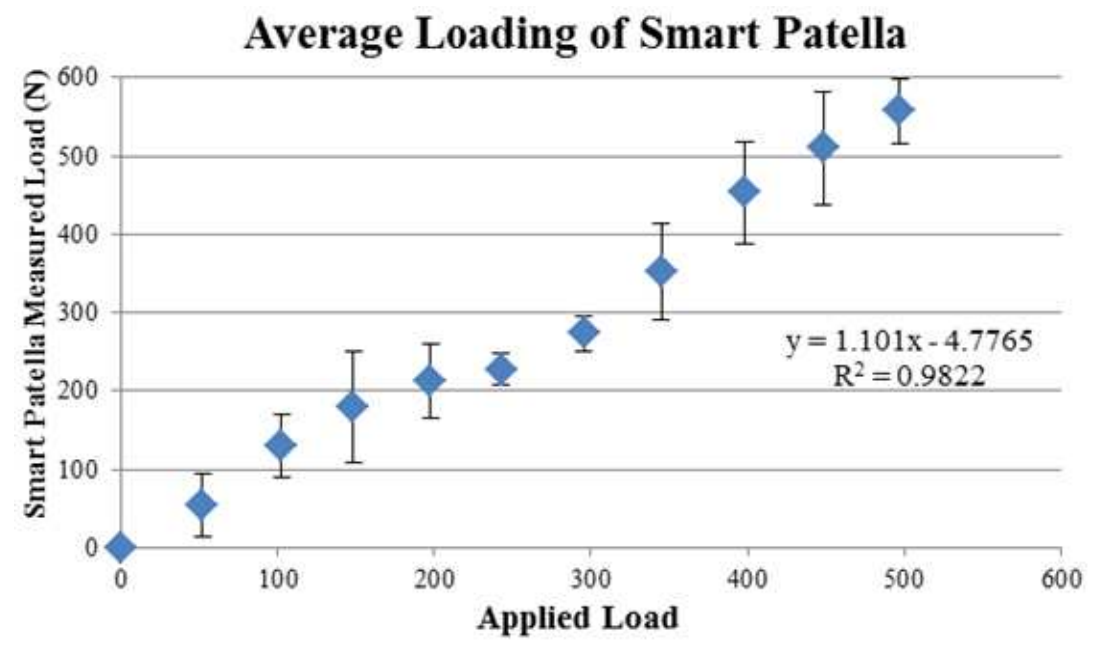

Fig. 7. Average force measurement was calculated and compared to applied load. Error bars represent one standard deviation

\section{Discussion}

In vivo, measuring forces across the patellofemoral joint has remained elusive. This is due to the characteristically small joint space and complex articulation. Traditional force measurement technologies (i.e., strain gauges and telemetry) cannot be adapted for this small space. Our novel force sensors are low profile, require no on-board electronics and can be interfaced with implants with no modification required. Results from this study demonstrate for the first time that our sensors can interface with a patellar button without modification and measure changes in force. Due to their design, the halo sensors can be placed around the posts on the anterior surface of the patellar button, as shown in Fig. 8, requiring no modification to the existing implant.
In this study, sensors were simultaneously interrogated throughout loading to characterize the changes in force magnitude and distribution across the patellofemoral joint. Sensors were individually calibrated in the range of $0-500 \mathrm{~N}$ which is the expected intraoperative force range for the patellofemoral joint (Singerman et al., 1999). Following individual testing, the sensors were placed in a smart patella arrangement, which allowed for the sensors to be simultaneously loaded and interrogated. From this testing, the data showed that all 3 sensors could be simultaneously interrogated wirelessly and that the error between measure load and applied load was small. Following calibration, force measurements with the smart patella were in excellent agreement with applied loads. This was further validated by the small mean error between applied load and forces measured by the smart patella which were $1.2 \mathrm{~N}$ over the $0-500 \mathrm{~N}$ loading range. 


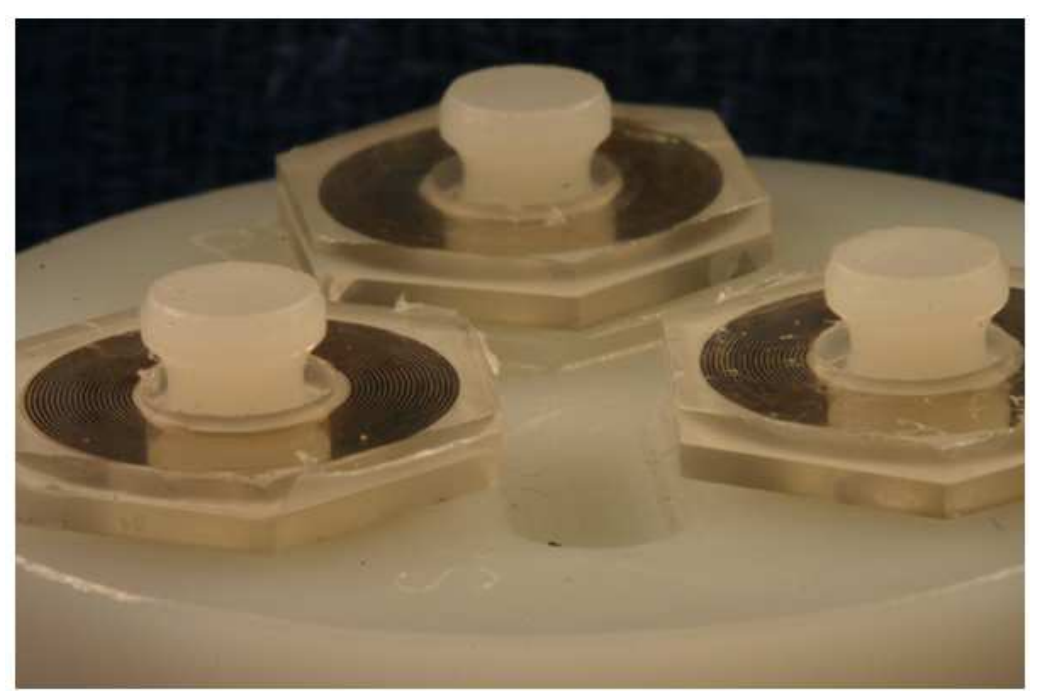

Fig. 8. Three halo sensors were placed around the posts on the anterior surface of a patellar implant with no modifications to the implant.

In this study, a novel smart patella was assembled and tested under axial loading. Results from this study indicate that the smart patellar implant can measure loads across a simulated patellofemoral joint. The mean error over the $0-500 \mathrm{~N}$ loading range was 12.2 N. Future testing will validate the performance of the smart patella in physiologically relevant conditions and through the entire range of expected in vivo forces $(0-4,000 \mathrm{~N})$.

\section{Conclusion}

This novel force sensing technology allows for accurate force measurement across a simulated patellofemoral joint. Results demonstrate for the first time that our novel force sensors can be interfaced with an off-the-shelf patellar button. Implementing these force sensors with off-the-shelf implants decreases the complexity and eliminates the re-design that has been necessary in other smart implant applications. By eliminating modifications, the structural integrity of the implant is not impacted and the clinical function of the implant is not diminished. Once interfaced with the patellar button, our force sensors enabled the characterization of force magnitude and distribution. Measuring force magnitude and distribution may lead to a better understanding of patellofemoral biomechanics and the mechanisms that lead to AKP, leading to a decrease in complications post-operatively.

\section{Funding Information}

This research was supported by the NYS NYCAP Research Alliance and by an institutional grant from the Scripps Health.

\section{Author's Contributions}

Matthew Dion: Experimental design, sensor fabrication, data collection and analysis, Manuscript preparation.

John Drazan, Sarah Giddings and Khaled Abdoun: Data collection and analysis.

Vishal Desai: Coil fabrication and design.

Nathaniel Cady: Coil fabrication and design.

Reena Dahle: Wireless communication and antenna design.

Jared Roberts: Study design and sensor design.

Eric Ledet: Principal investigator. Study design, data analysis and interpretation, Manuscript preparation.

\section{Conflict of Interest}

Eric Ledet is a co-founder, officer and stakeholder in inSense Medical LLC and I/O Surgical.

\section{References}

Drazan, J.F., A. Gunko, M.K. Dion, O. Abdoun and N.C. Cady et al., 2014. Archimedean spiral pairs with no electrical connections as a passive wireless implantable sensor. J. Biomed. Technol. Res.

Kurtz, S., K. Ong, E. Lau, F. Mowat and M. Halpern, 2007. Projections of primary and revision hip and knee arthroplasty in the United States from 2005 to 2030. J. Bone Joint Surgery, 89: 780-5. DOI: 10.2106/00004623-200704000-00012

Losina, E., R.P. Walensky, C.L. Kessler, P.S. Emrani and W.M. Reichmann et al., 2009. Costeffectiveness of total knee arthroplasty in the United States. Arch. Internal Med., 169: 1113-1121. DOI: 10.1001/archinternmed.2009.136 
Metsna, V., S. Vorobjov and A. Märtson, 2014. Prevalence of anterior knee pain among patients following total knee arthroplasty with nonreplaced patella: A retrospective study of 1778 knees. Medicina, 50: 82-6.

DOI: 10.1016/j.medici.2014.06.001

Petersen, W., I.V. Rembitzki, G.P. Brüggemann, A. Ellermann and R. Best et al., 2014. Anterior knee pain after total knee arthroplasty: a narrative review. Int. Orthopaedics, 38: 319-28. DOI: $10.1007 / \mathrm{s} 00264-013-2081-4$

Sensi, L., R. Buzzi, F. Giron, L. De Luca and P. Aglietti, 2011. Patellofemoral function after total knee arthroplasty: Gender-related differences. J. Arthroplasty, 26: 1475-80.

DOI: $10.1016 /$ j.arth.2011.01.016
Singerman, R., S.M. Gabriel, C.B. Maheshwer and J.W. Kennedy, 1999. Patellar contact forces with and without patellar resurfacing in total knee arthroplasty. J. Arthroplasty, 14: 603-9. DOI: 10.1016/S0883-5403(99)90084-0

Wachs, R.A., D. Ellstein, J. Drazan, C.P. Healey and R.L. Uhl et al., 2013. Elementary implantable force sensor: For smart orthopaedic implants. Adv. Biosensors Bioelectron., 2: 12477-12477. PMID: 24883335

Zou, Y., Z. Chen, Z. Feng and J. Xing, 2011. Factors related to anterior knee pain after total knee arthroplasty. J. Southern Med. Univ., 31: 1428-1430. PMID: 21868338 